\title{
YouTube as a Health Information Source: COVID-19 and Andrology
}

\author{
๑ Murat Demir, ๑ Kerem Taken, ๑ Recep Eryilmaz, @ Rahmi Aslan, ๑ Kasim Ertas \\ Van Yuzuncu Yıl University Faculty of Medicine, Department of Urology, Van, Turkey
}

\section{Abstract}

\begin{abstract}
Aim: The purpose of this study is to evaluate the quality and reliability of YouTube videos about infertility, erectile dysfunction and sex relationship with coronavirus disease-2019 (COVID-19).

Methods: In this prospective study, videos were selected on 20.12.2020 and variable parameters were studied on the same day. Nonvariable parameters such as quality and reliability were studied until 25.12.2020. "Corona, infertility", "corona, erectile dysfunction", "corona, sex", "COVID, infertility", "COVID, erectile dysfunction", COVID, sex", "pandemic, infertility" "pandemic, erectile dysfunction" and "pandemic, sex" search words scanned in a private search mode on the YouTube website. Unrelated, not English, do not contain any information videos excluded. The remaining videos were evaluated according to the presenter source and the presented audience with modified discern and Global Quality Scala (GQS) forms.
\end{abstract}

Results: Fourteen (14\%) of 100 videos were about erectile dysfunction, 56 (56\%) were about infertility and 30 (30\%) were about sex. Fifteen (15\%) of the videos were presented by individual sources, 4 (4\%) by non-physician healthcare professionals, 48 (48\%) by individual physicians, $17(17 \%)$ by physician groups, $13(13 \%)$ by news agencies, and $3(3 \%)$ by private companies. While $87(87 \%)$ of the target group was the general public, $13(13 \%)$ of them were healthcare professionals. According to the presenter source, the modified discern and GQS scores of the physician group were higher than the other groups. However, the number of views and likes in these two groups was low.

Conclusion: Videos offered by physician groups and targeted by healthcare professionals on YouTube are of higher quality and more reliable. However, the popularity of videos is not a suitable indicator of quality.

Keywords: Social media, infertility, erectile dysfunction

\section{Introduction}

The new coronavirus disease-2019 (COVID-19) epidemic that started in Wuhan, China in December 2019 caused a serious health crisis worldwide in a short time (1). This infection, which was declared a pandemic by the World Health Organization on March 11 2020, has now caught more than 80 million people and nearly two million people have died (1). Despite social distancing and isolation measures, the number of cases is still increasing.

Pneumonia is the most common complication of COVID-19 (2). However, many studies have shown that other organs are also affected (3).

Andrologically, studies have indicated that the virus can cause erectile dysfunction and infertility $(4,5)$. However, most of these studies are not yet strongly proven. However, such information has led to speculation on websites, news programs, and especially on social media, and has made people nervous (6). It has been stated that incomplete or incorrect information on social media is one of the most damaging issues to fight COVID-19 (7). It is possible that failure to inform the society completely and correctly can cause social chaos and panic (8). Therefore, it is important to filter information sources in this global crisis.

YouTube, which we used in our study, is the largest video site in the world and the second largest search page. This site has more than one billion users and hundreds of millions of videos $(9,10)$. However, since videos do not pass any filter on this site, false and harmful information is shared as well as true information. In a study on breast cancer, most of the YouTube videos have misrepresented self-examination and by explaining that breast cancer is

Address for Correspondence: Murat Demir, Van Yuzuncu YIl University Faculty of Medicine, Department of Urology, Van, Turkey 
seen only in women, it has been observed that there are false and harmful directions (11). YouTube videos on Zika and Ebola infections from previous public health crises have been viewed millions of times. However, studies have shown that most of the videos are misleading $(12,13)$. For these reasons, it is important to evaluate videos in terms of quality and reliability.

In this study, the videos shared on the YouTube website about infertility, erectile dysfunction and sexuality relationship with COVID-19 were evaluated in terms of quality and reliability.

\section{Methods}

In this prospective study, after the approval of the local ethics committee (dated 01.12.2020 and numbered 2020/09-22), the videos were selected on 20.12.2020. At the same time, the number of likes, dislikes, views and comments, which are the variable data of the videos, was studied. The unvariable parameters (quality and reliability) were studied until 25.12.2020. On the YouTube website "corona, infertility", "corona, erectile dysfunction", "corona, sex", "COVID, infertility", "COVID, erectile dysfunction", COVID, sex" the keywords "pandemic, infertility" "pandemic, erectile dysfunction" and "pandemic, sex" were searched. The searches were made in incognito mode in the cache-cleared web browser where all updates were available. The upload date from the filters was adjusted according to "this year" and the ranking criterion relevance. Videos with keywords in the video title were included in the study.

After searching, 152 videos were evaluated. Videos that were repeated, non-English, irrelevant, or did not contain any audio or visual information were excluded.

The remaining 100 videos after these exclusion criteria were evaluated separately by two expert urologists (MD, $\mathrm{RE}$ ). Reliability among experts was calculated by kappa statistics. When no consensus was reached, a third expert (RA) was consulted.

The duration of the videos included in the study, the number of views, upload time, number of comments, likes and dislikes were recorded. The videos were evaluated according to host sources and target audiences. Server sources were categorized into 6 groups as individual, non-physician healthcare provider, individual physician, physician group, journalist and private company.

The target audiences were categorized as healthcare professionals and the general audience.

Video topics were divided into 3 as erectile dysfunction, infertility, sex (transmission during sex, protection, sexual interference, etc.).

The Global Quality Score (GQS) form was used to evaluate the overall quality of the videos. This form consists of five degrees that question the quality, flow, information content and usefulness of the videos. GQS: 1 indicates poor quality, while GQS: 5 shows excellent quality (14).

Discern short modified form was used to evaluate the reliability of the videos. It is a modified version of the form developed by Modified Discern Charnock and Shepperd (15). In this form with a 5-rating, videos with 3 points are considered medium quality, while those who score more than 3 are considered quality, areas less than 3 are considered low quality.

\section{Statistical Analysis}

Descriptive statistics for continuous variables; it is expressed as average, standard deviation, minimum and maximum values, and expressed as numbers and percentages for categorical variables. One-way analysis of variance was used to compare group averages in terms of continuous variables. Following the variance analysis, Duncan multiple comparison test was used to identify different groups. Pearson correlation coefficients were calculated to determine the relationship between these variables. Chi-square test was used to determine the relationship between the groups and categorical variables. The statistical significance level was taken as $5 \%$ in the calculations and the SPSS statistical package program was used for calculations.

\section{Results}

After 152 videos were evaluated according to exclusion criteria, the remaining 100 videos were included in the study. The average upload time of the videos is $224.43 \pm 197$ days, the length is $859.05 \pm 1.313 \mathrm{sec}$, the number of likes is $293.49 \pm 1110.55$, the dislike is $112.14 \pm 477.60$, the number of views is $26586.8 \pm 74207.1$, and the number of comments is $10.8 \pm 397.94$. The kappa coefficient among experts (MD, RE) was 0.91 .

While there were $14(14 \%)$ videos about erectile dysfunction, 56 (56\%) were about infertility, and 30 (30\%) were about sex. According to the topics of videos, the groups were homogeneous regarding length, number of likes and dislikes, views, comments, Discern and GQS results. Fifteen (15\%) of the videos were individual sources, 4 (4\%) non-physician healthcare professionals, $48(48 \%)$ individual physicians, $17(17 \%)$ physicians, 13 $(13 \%)$ news agencies, $3(3 \%)$ of them were offered by private firms. The properties of the videos by the server source are listed in Table 1.

While $87(87 \%)$ of the target audience in the videos was the public, $13(13 \%)$ were healthcare professionals. Considering the target audience of the videos, the duration of the videos made for healthcare professionals was significantly longer $(p=0.001)$. However, there was no significant difference in terms of upload time, likes, 
dislikes, views and comments of the videos. Videos with GQS 5 were significantly longer than other videos $(p=0.001)$. While there was no significant difference in the number of likes according to the GQS values, the GQS 5 was significantly lower in the dislike numbers $(p=0.02)$.

The number of views was highest in GQS 2 with an average of $71421.20(p=0.02)$. There was no statistically significant difference in the number of comments compared to the GQS values. GQS scores according to the video source and target audience are in Table 2,3. According to the server, the Discern score was significantly higher in the physician group and compared to the target group in the healthcare group (Table 4).

\section{Discussion}

Today, the Internet has become a platform where information about health can be easily obtained. More than $80 \%$ of Americans use the Internet to obtain health information (16). The Internet is an easily accessible tool to obtain information on issues that have become taboo in society, especially sexual and reproductive health $(17,18)$.

YouTube is the most popular video website with over 1 billion users and millions of videos. In a study conducted on male infertility, it was observed that Twitter, one of the social media platforms, refers to YouTube videos with the hashtag \#maleinfertility and online traffic is directed to YouTube (19). In the study by Fode et al. (20), they showed that every month millions of people watch erectile dysfunction videos on YouTube.

In addition, according to YouTube data, 95\% of Internet users use YouTube (21). For these reasons, the results we obtained are generalizable.

It was seen that most of the videos about the relationship between COVID-19 and ED, infertility and sexual health was shared by individual physicians, targeting the public. The highest scores in terms of the

\begin{tabular}{|c|c|c|c|c|c|c|c|}
\hline & $\begin{array}{l}\text { Individual } \\
\text { sources }\end{array}$ & $\begin{array}{l}\text { Non-physician } \\
\text { healthcare } \\
\text { professionals }\end{array}$ & $\begin{array}{l}\text { Individual } \\
\text { physicians }\end{array}$ & $\begin{array}{l}\text { Physician } \\
\text { groups }\end{array}$ & $\begin{array}{l}\text { News } \\
\text { agencies }\end{array}$ & $\begin{array}{l}\text { Private } \\
\text { companies }\end{array}$ & $p$ \\
\hline Number of views & 49402.27 & 3834.75 & 13863.94 & 341.35 & 94627.08 & 294.67 & $0.004^{*}$ \\
\hline Like & 376.80 & 98.00 & 135.35 & 3.18 & 1286.62 & 9.33 & $0.02^{*}$ \\
\hline Dislike & 35.73 & 52.75 & 44.85 & 0.12 & 639.15 & 1.0 & $0.002^{*}$ \\
\hline Comments & 73.47 & 10.25 & 77.14 & 0.82 & 432.23 & 0.67 & 0.053 \\
\hline Upload time & 136.27 & 159.50 & 181.94 & 147.94 & 117.00 & 164.00 & 0.92 \\
\hline Daily viewing & 362.5 & 24.04 & 76.19 & 2.30 & 808.17 & 1.79 & 0.001 * \\
\hline Video duration & 598.60 & 1193.75 & 464.00 & 2795.24 & 168.62 & 56.00 & $0.001 *$ \\
\hline
\end{tabular}

\begin{tabular}{|c|c|c|c|c|c|c|}
\hline Target group & GQS 1 & GQS 2 & GQS 3 & GQS 4 & GQS 5 & total \\
\hline General & 11 & 20 & 29 & 19 & 8 & 87 \\
\hline Physician & 0 & 0 & 0 & 7 & 6 & 13 \\
\hline Total & 11 & 20 & 29 & 26 & 14 & 100 \\
\hline
\end{tabular}

\begin{tabular}{|c|c|c|c|c|c|c|c|}
\hline \multirow{2}{*}{ Video Source } & \multicolumn{5}{|l|}{ GQS } & \multirow[t]{2}{*}{ Total } & \multirow{9}{*}{$p<0.001^{*}$} \\
\hline & GQS 1 & GQS 2 & GQS 3 & GQS 4 & GQS 5 & & \\
\hline Individual sources & 4 & 9 & 2 & 0 & 0 & 15 & \\
\hline Non-physician healthcare professionals & 0 & 0 & 1 & 2 & 1 & 4 & \\
\hline Individual physicians & 0 & 4 & 24 & 16 & 4 & 48 & \\
\hline Physician groups & 0 & 0 & 0 & 8 & 9 & 17 & \\
\hline News agencies & 5 & 6 & 2 & 0 & 0 & 13 & \\
\hline Private companies & 2 & 1 & 0 & 0 & 0 & 3 & \\
\hline Total & 11 & 20 & 29 & 26 & 14 & 100 & \\
\hline
\end{tabular}


Table 4. Discern score according to the target audience

\begin{tabular}{|l|l|l|}
\hline & Discern score & $\mathbf{p}(\boldsymbol{\chi} \mathbf{2})$ \\
\hline & Server resource & \\
\hline Individual sources & $1.3 \pm 0.48$ & - \\
\hline Non-physician healthcare professionals & $1.75 \pm 0.5$ & - \\
\hline Individual physicians & $2.6 \pm 0.49$ & $\mathrm{p}<0.05^{*}$ \\
\hline Physician groups & $3.35 \pm 0.7$ & - \\
\hline News agencies & $1.3 \pm 0.47$ & - \\
\hline Private companies & $1.3 \pm 0.50$ & - \\
\hline & Target Audience & \\
\hline General population & $2.14 \pm 0.84$ & $\mathrm{p}<0.05^{*}$ \\
\hline Healthcare professionals & $3.30 \pm 0.63$ & - \\
\hline (According to theservin &
\end{tabular}

(According to the server resource; the physician group, and according to the target audience; the healthcare professionals are significantly higher) (chi-square- $\chi 2$ )

quality and usefulness of the videos were the videos of the physician group. It is natural that there is more than one physician as a scientific resource in the physician group, and scientific quality increases due to the exchange of ideas and the transformation of speech into a scientific environment. Despite this, Discern and GQS scores of both physician groups and individual physicians were not at a satisfactory level in our study. The reason for this may be that the relationship between COVID-19 and andrological diseases is dark, the literature is inadequate, and there is no consensus among international health organizations. In the study in which Culha et al. (22) evaluated YouTube videos about pelvic floor muscle exercises, it was seen that the quality and reliable information was shared by healthcare professionals (doctors, nurses, physiotherapists) regardless of the branch. In addition, the Discern scores of the videos presented by healthcare professionals were higher than our study. This may be due to the general lack of knowledge between COVID-19 and andrological diseases. In the same study, healthcare providers obtained the highest GQS scores in line with our study (22). In addition, when the target audience in our study was healthcare professionals, it is thought that the increase in both the quality and reliability of the videos is because the information is given with more detailed and literature information.

In a study evaluating erectile dysfunction videos on YouTube, it was observed that the videos provided by groups of healthcare professionals were of higher quality than individual professionals and nonprofessional groups, in accordance with our study (13). Similar results were also obtained in a study on laryngeal cancer (23). Ku et al. (24) stated that YouTube videos can be useful for informing about infertility, but high-quality videos are needed. In the field of urology, Fode et al. (20) found the videos suspicious in their study, where they evaluated videos on erectile dysfunction on YouTube (20). In a study by Gul and Diri (25), they stated that there were many misleading videos about prematüre ejeculation on YouTube and they suggested that they should be removed. In our study, although the Discern and GQS scores were higher in the videos presented by the physician groups, it was still not at the desired level. In addition, these scores decrease even more in groups other than the physician group. These data also show that YouTube videos are not reliable and of sufficient quality.

In our study, it was observed that high-quality and reliable videos had a small number of views and likes. In another study on YouTube videos about COVID-19 and pregnancy relationship, it was observed that news agencies were the most watched videos, but their discern scores were low (26). Therefore, popularity does not seem to be a suitable criterion for predicting video quality. However, according to the results of our study, the number of dislikes can be a criterion in terms of reliability and quality.

\section{Study Limitations}

Our working together with all of these has some limitations. Since YouTube has a dynamic structure, new videos can be added and removed constantly, and the number of views, likes and dislikes can change. However, as only English language videos were used in our study, it had a relatively small sample. It should be kept in mind that although the video searches are made in incognito mode with the cache cleared, our geographic location and computer language may affect the search results.

\section{Conclusion}

As a result, although YouTube website has an important source of information in terms of COVID-19 and andrological problems, it is important to be careful in terms of the reliability and quality of the videos. It should also be known that non-physician server resources are unreliable enough.

\section{Authorship Contributions}

Concept: M.D., K.T., K.E., Design: M.D., K.T., R.E., R.A., K.E., Data Collection or Processing: M.D., K.T., R.E., R.A., K.E., Analysis or Interpretation: M.D., K.T., R.E., R.A., K.E., Literature Search: M.D., K.T., R.E., R.A., K.E., Writing: M.D., R.A., R.E.

Conflict of Interest: No conflict of interest was declared by the authors.

Financial Disclosure: The authors declared that this study received no financial support.

\section{References}

1. Shi $H$, Han X, Zheng C. Evolution of CT Manifestations in a Patient Recovered from 2019 Novel Coronavirus (2019-nCoV) Pneumonia in Wuhan, China. Radiology 2020;295:20.

2. Fang $Y$, Zhang $H$, Xie J, et al. Sensitivity of Chest CT for COVID-19: Comparison to RT-PCR. Radiology 2020;296:115-7. 
3. Yıldırım Ö, Turgay A, Tunay D. COVID-19 Pandemisi ve Kardiyovasküler Etkileri. Çukurova Anestezi ve Cerrahi Bilimler Dergisi 2020;3:128-33.

4. Sansone A, Mollaioli D, Ciocca G, et al. Addressing male sexual and reproductive health in the wake of COVID-19 outbreak. J Endocrinol Invest 2021;44:223-31.

5. Aitken RJ. COVID-19 and human spermatozoa-Potential risks for infertility and sexual transmission? Andrology 2021;9:4852.

6. Pennanen-lire C, Prereira-Lourenço M, Padoa A, et al. Sexual Health Implications of COVID-19 Pandemic. Sex Med Rev 2021;9:3-14.

7. Şeker M, Özer A, Tosun Z, Korkut C, Doğrul M. Covid-19 Pandemi Değerlendirme Raporu: Türkiye Bilimleri Akademisi 2020;1-164.

8. Kırık AM, Özkoçak V. Yeni Dünya Düzeni Bağlamında Sosyal Medya ve Yeni Koronavirüs (COVID-19) Pandemisi. SOBIDER 2020;7:133-154.

9. Cheng X, Dale C, Liu J. Statistics and social network of Youtube videos. 16th Interntional Workshop on Quality of Service 2008;229-38.

10. Chatzopoulou G, Sheng C, Faloutsos M. A first step towards understanding popularity in YouTube.In: 2010 INFOCOM IEEE Conference on Computer Communications Workshops 2010;1-6.

11. Esen E, Aslan M, Sonbahar BÇ, Kerimoğlu RS. YouTube English videos as a source of information on breast self-examination. Breast Cancer Res Treat 2019;173:629-35.

12. Bora K, Das D, Barman B, Borah P. Are internet videos useful sources of information during global public health emergencies? A case study of YouTube videos during the 2015-16 Zika virus pandemic. Pathog Glob Health 2018;112:320-8.

13. Basch $\mathrm{CH}$, Basch $\mathrm{CE}$, Ruggles $\mathrm{KV}$, Hammond R. Coverage of the Ebola Virus Disease Epidemic on YouTube. Disaster Med Public Health Prep 2015;9:531-5.

14. Ferhatoglu MF, Kartal A, Ekici U, Gurkan A. Evaluation of the Reliability, Utility, and Quality of the Information in Sleeve Gastrectomy Videos Shared on Open Access Video Sharing Platform YouTube. Obes Surg 2019;29:1477-84.

15. Borgmann H, Wölm JH, Vallo S, et al. Prostate Cancer on the Web-Expedient Tool for Patients' Decision-Making? J Cancer Educ 2017;32:135-40.
16. Chen Y, Li C, Liang J, Tsai C. Health information obtained from the Internet and changes in medical decision making: questionnaire development and cross-sectional survey. Journal of Medical Internet Research 2018;20:47.

17. Lorenc A, Robinson N. A tool to improve patient and public engagement in commissioning sexual and reproductive health and HIV services. J Fam Plann Reprod Health Care 2015;41:8-12.

18. Gabarron E, Wynn R. Use of social media for sexual health promotion: a scoping review. Glob Health Action 2016;9:32193.

19. Balasubramanian A, Yu J, Thirumavalavan N, Lipshultz LI, Hotaling JM, Pastuszak AW. Analyzing online twitter discussion for male infertility via the hashtag \#Malelnfertility. Urol Pract 2020;7:68-74.

20. Fode M, Nolsøe AB, Jacobsen FM, et al. Quality of Information in YouTube Videos on Erectile Dysfunction. Sex Med 2020;8:408-13.

21. YouTube by the Numbers: Stats, Demographics. Available from: https://www.businessofapps.com/data/youtubestatistics/. Accessed 2020 May 15.

22. Culha Y, Seyhan Ak E, Merder E, Ariman A, Culha MG. Analysis of the YouTube videos on pelvic floor muscle exercise training in terms of their reliability and quality. Int Urol Nephrol 2021;53:1-6.

23. Enver N, Doruk C, Kara H, Gürol E, Incaz S, Mamadova U. YouTube $^{\mathrm{TM}}$ as an information source for larynx cancer: a systematic review of video content. Eur Arch Otorhinolaryngol 2020;277:2061-9.

24. Ku S, Balasubramanian A, Yu J, et al. A systematic evaluation of youtube as an information source for male infertility. Int J Impot Res 2020 Jun 15.

25. Gul M, Diri MA. YouTube as a Source of Information About Premature Ejaculation Treatment. J Sex Med 2019;16:173440.

26. Yuksel B, Cakmak K. Healthcare information on YouTube: Pregnancy and COVID-19. Int J Gynaecol Obstet 2020;150:189-93. 